\title{
Development of a Multiple Degree of Freedom Knee Disarticulation Prosthesis with Active Leg Length Variation
}

\author{
Berend Denkena, Martin Eckl, Dominik Brouwer \\ Leibniz Universität Hannover, Institute of Production Engineering and Machine Tools, An der Universität 2, \\ Hanover, Germany \\ Email: Denkena@ifw.uni-hannover.de, Eckl@ifw.uni-hannover.de, Brouwer@ifw.uni-hannover.de
}

Received 2 September 2014; revised 21 October 2014; accepted 6 November 2014

Copyright @ 2014 by authors and Scientific Research Publishing Inc.

This work is licensed under the Creative Commons Attribution International License (CC BY). http://creativecommons.org/licenses/by/4.0/

c) (i) Open Access

\section{Abstract}

This paper presents a novel design for a knee disarticulation prosthesis. In this design, three hydraulic cylinders form the supporting structure and provide the damping effect at the same time. That way the novel knee joint offers two fundamental advantages compared to the state of the art. First, the combination of a supporting structure and damping element reduces the weight of the prosthesis. Secondly, the use of several cylinders allows the actuation of further degrees of freedom. Additional degrees of freedom can be used to vary the leg length within the gait cycle and hence to optimize the gait behavior.

\section{Keywords}

Biomechanics, Biomedicine, Prostheses, Knee-Disarticulation

\section{Introduction}

Exoprostheses are orthotics to balance functionality and cosmetic aspects of lost extremities. In the past 100 years the limb amputation indication changed from life saving measures due to injury to plastic, micro surgical intervention. The indication and the individual height of amputation influence the rehabilitation course. Hagberg et al. show that the higher the level of amputation, the lower the degree of rehabilitation; hence a below knee amputation should be aspired [1]. Figure 1 shows the different amputation heights.

If a higher level of amputation is not avoidable, knee disarticulation is to be considered rather than above knee amputation. Knee disarticulation was the amputation through the knee joint and was performed first in 1825 in the USA [2]. Compared to above knee amputation, knee disarticulation offers many advantages. The knee stump 


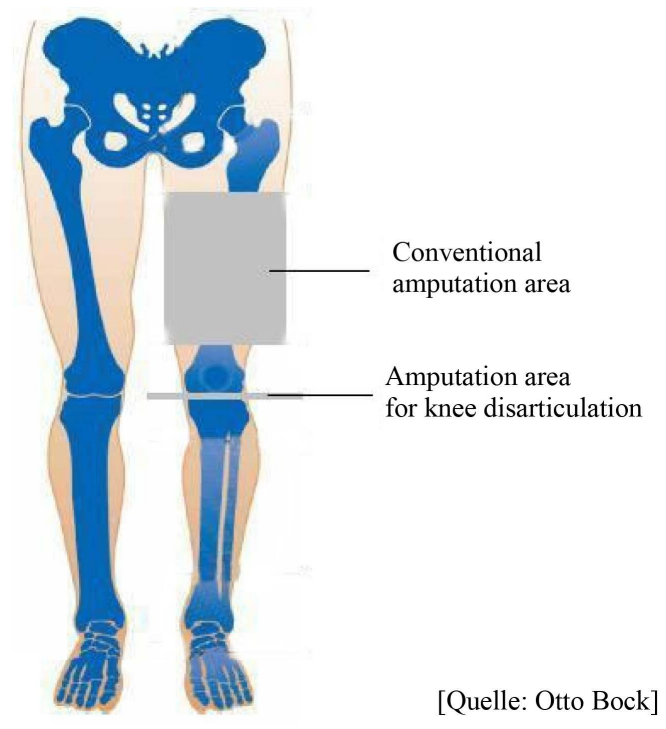

Figure 1. Schematic representation of the different amputation levels.

keeps the ability to absorb the weight force as the ischial tuberosity is spared [3]. Furthermore, the knee disarticulation does not affect the femur, and patella [4]. Thus, the remaining thigh muscles allow for coordinated stump movement [5]. In case of knee disarticulation, many different designs of the artificial knee joint can be applied. Stark gives a brief overview of such different designs [6]. In principle, any prosthesis for above knee amputees is suitable for knee disarticulation amputees as well. However, as the natural knee stump is still present, the artificial knee joint would be placed below the natural knee. These different anatomic situations need to be considered when selecting a knee joint design.

Current artificial knee joints are designed as single axis or polycentric joints. When single axis joints are used for knee disarticulation amputees, the distance between the anatomic and the artificial knee pivot point is long. This leads to an unnatural gait behavior and less stability within the walking cycle. O'Connor introduces a single axis joint where a special knee bracket reduces the distance between the anatomic knee and the pivot of the artificial knee and provides a more naturally looking and operating prosthetic leg [7]. In contrast to single axis joints, polycentric knee joints are mainly used for knee disarticulation amputees. In this case, the pivot is variable and outside of the actual knee joint mechanism. The design of such polycentric knee joints can be optimized to the requirements of knee disarticulation amputees. The University of Toledo has developed a method to optimize a polycentric knee joint for knee disarticulation prostheses [8]. All current knee joints consist of a mechanical supporting structure and an additional damping element.

In contrast, this paper presents a novel design for a polycentric knee joint for knee disarticulation prostheses, where hydraulic cylinders form the supporting structure and provide the damping effect at the same time (Figure 2).

The novel knee joint offers two fundamental advantages compared to the state of the art. First, the combination of a supporting structure and damping element reduces the weight of the prosthesis. Secondly, the use of several cylinders allows the actuation of further degrees of freedom. Additional degrees of freedom can be used to vary the leg length within the gait cycle and hence to optimize the gait behavior. The patented design [9] was developed within the cooperative research project MultiPro, funded by the Federal Ministry for Research and Education (BMBF).

This paper is organized as follows. Section 2 explains the design and the functionality of the named knee joint mechanism, followed by the control scheme. Further, a test rig is presented, which is able to simulate a gait cycle. Section 3 discusses the mathematical relations between the mechanical and the hydraulic part of the knee joint. Based on these mathematical relations, a control scheme is developed to absorb the torque, generated by the weight of the patient and the lever arm between the line of action of the weight force and the prosthesis. Furthermore, a simulation model of the mechanism is developed using the software tools SimMechanics and 


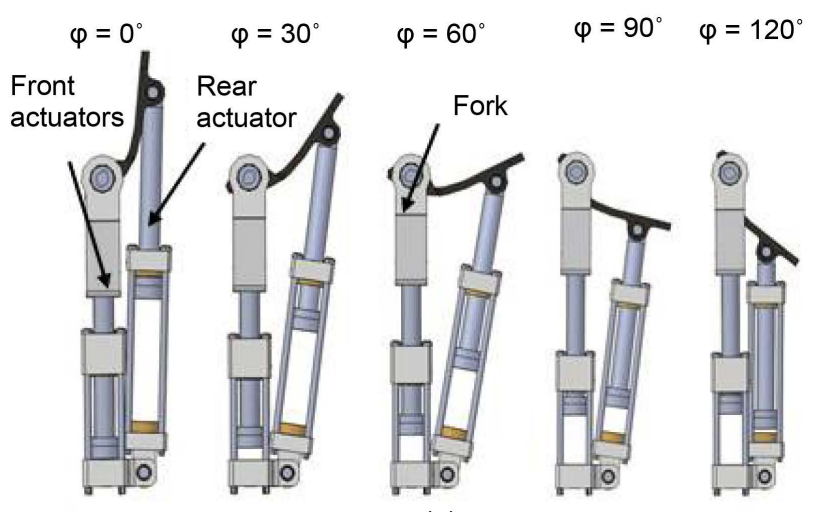

(a)
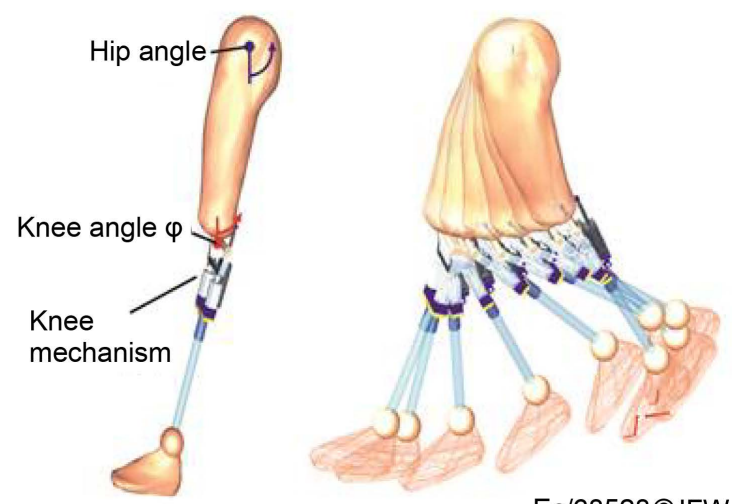

(b)

Ec/68528ㅇIFW

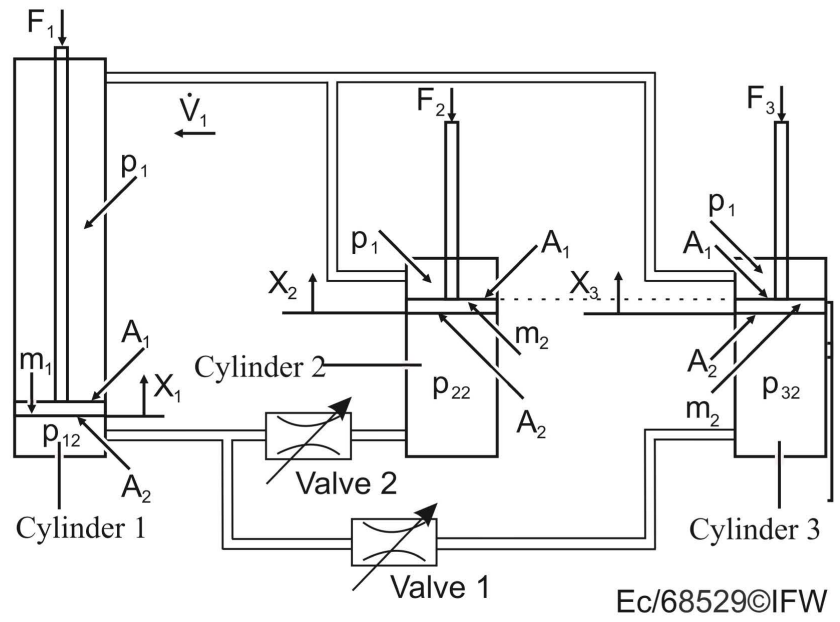

(c)

Figure 2. Design of the knee mechanism. (a) CAD model for different knee angles; (b) Knee mechanism within a gait cycle; (c) Hydraulic system overview.

SimHydraulics (Mathworks). In Section 4 the hydraulic scheme is extended to enable active leg length variation within the gait cycle. The control scheme is simulated using Matlab/Simulink. That enables the simulation of a whole gait cycle with leg length variation. Finally, the development results of the research project are summarized in the conclusion.

\section{Design of the Knee Mechanism}

\subsection{Description of the Novel Knee Mechanism}

Figure 2(a) shows the CAD model of the artificial knee mechanism for different knee angles. The knee mechanism consists generally of three linear hydraulic actuators. Two of the actuators are arranged symmetrically in the front of the prosthesis, while the third is arranged at the back of the prosthesis. Figure 2(b) illustrates the novel knee mechanism and a leg within the gait cycle (the kinematics of a gait cycle is shown in Figure 2). The left-top part of Figure 2 depicts how different angles are realized with the novel knee mechanism. To realize the knee of zero degrees, the front cylinders are at zero stroke and the back cylinder is at maximum stroke. To realize an angle, the forward cylinders are extended, the rearward cylinder is retracted. In this way, angles up to $120^{\circ}$ can be realized. If the front cylinders and the rear cylinder are retracted, the length of the prosthesis is shortened, if all actuators are extended, the prosthesis is extended accordingly.

Figure 2(c) shows the hydraulic circuit diagram. The linear actuators are realized as hydraulic cylinders in a closed system. The volume of two front cylinders (indices 2 and 3) is equal to the volume of the rear cylinder (index 1). In this way, only the desired defined knee-angles can be realized. To implement a damping functionality, two electronically controllable throttle valves are integrated. To further implement the functionality of 
controlled leg length change, additional components have to be included. These components are described in Section 4. The change of the knee angle is driven by the external influences: hip movement, the patient weight force, and the ground reaction force. In the swing phase, the hip is moved forward and, thus, because of the inertia of the prosthesis, the prosthesis leg. In the stance phase the foot is fixed at the ground and the body is moved forward mainly by the hip movement. The ground reaction force and the movement of the hip generate a torque at the fork. So, in both phases of the gait, a counter direction movement of the rearward cylinder (cylinder 1) and the forward cylinders (cylinders 2 and 3) is generated. The fluid flows through the proportional valves 1 and 2 and the knee angle can be damped by adjusting the cross section of the valves to generate a sufficient torque in the stance phase and to control the correct knee angle in the swing phase. The damping value is set by proportional valves, depending on the current gait phase.

The invented knee mechanism is designed to sustain the mechanical stress, required by standard ISO10328 [10]. Most parts have been optimized using the Finite Element Method. If possible, lightweight materials have been used. The parts of the prosthesis, their materials and the corresponding mass are shown in Table 1 . The knee mechanism has a weight of $1.115 \mathrm{~kg}$ while the whole prosthesis weighs $2.5 \mathrm{~kg}$ and is designed for patients with a maximum weight of $125 \mathrm{~kg}$. A maximum knee angle of $136^{\circ}$ is attainable.

\section{Table 1. Overview of the components and its weights used in the knee joint.}
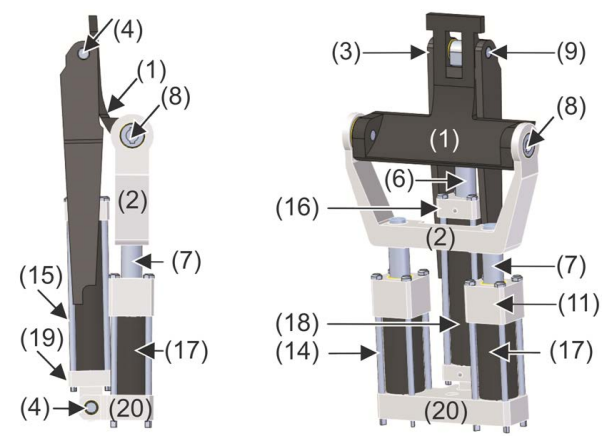

\begin{tabular}{ccccc}
\hline Item & Qty & Component & Material & Weight \\
\hline 1 & 1 & Socket adapter & CFRP & $78 \mathrm{~g}$ \\
2 & 1 & Suspension fork & Aluminum & $132 \mathrm{~g}$ \\
3 & 1 & Brace & CFRP & $72 \mathrm{~g}$ \\
4 & 2 & Joint bolt (rearward) & Stainless Steel & $16 \mathrm{~g}$ \\
5 & 4 & Bearing bushing (8 mm) & Iglidur-W300 & $0.5 \mathrm{~g}$ \\
6 & 1 & Piston and piston rod (rearward) & Stainless Steel & $99 \mathrm{~g}$ \\
7 & 2 & Piston and piston rod (forward) & Stainless Steel & $109 \mathrm{~g}$ \\
8 & 2 & Joint bolt (forward) & Stainless Steel & $10 \mathrm{~g}$ \\
9 & 2 & Screw M6×25 (DIN 7984) & Stainless Steel & $8 \mathrm{~g}$ \\
10 & 2 & Bearing bushing (15 mm) & Iglidur-W300 & $0.5 \mathrm{~g}$ \\
11 & 2 & Cylinder cover (forward) & Aluminum & $33 \mathrm{~g}$ \\
12 & 2 & Bearing bushing (13 mm) & Iglidur-W300 & $0.5 \mathrm{~g}$ \\
13 & 6 & Piston end stop & PV & $1 \mathrm{~g}$ \\
14 & 8 & Tension rod (forward) & Stainless Steel & $10 \mathrm{~g}$ \\
15 & 4 & Tension rod (rearward) & Stainless Steel & $12 \mathrm{~g}$ \\
16 & 1 & Cylinder cover (rearward) & Aluminum & $17 \mathrm{~g}$ \\
17 & 2 & Cylinder tube (forward) & CFRP & $26 \mathrm{~g}$ \\
18 & 1 & Cylinder tube (rearward) & CFRP & $44 \mathrm{~g}$ \\
19 & 1 & Cylinder bottom (rearward) & Aluminum & $23 \mathrm{~g}$
\end{tabular}




\subsection{Controlling a Gait Cycle}

The required damping value depends on the gait phase. During the stance phase, the knee has to provide enough resistance to generate a sufficient torque to accommodate the weight force of the patient. While in the swing phase, the damping has to be low but adaptive to generate a natural looking swinging movement. Thus, controlling a gait cycle requires the knowledge of the ground reaction forces in the stance phase, the current angle of the knee in the swing phase, and the desired movement of the knee. Any patient has his own pattern of movement, depending on the age, gender, body dimensions and, body proportions. Nevertheless, the general course of movement is nearly equal for any patient [11] [12] and called gait cycle. The kinematics of the knee and the hip joint within the gait cycle are depicted in Figure 3.

The shown cycle begins with the stance phase. In this phase, the hip is at around thirty degrees, the knee is strait, and the foot touches the ground. While the foot is touching the ground, the hip is rotated back to approx. zero degrees and the knee is bended to approx. eighteen degrees. In this phase, the body is moved forward. In the swing phase of the illustrated leg (the other leg would conduct the stance phase), the hip is rotated to the starting position, while the knee has to be rotated to around sixty degrees to avoid collision of the toes with the ground.

The gait cycle begins with the stance phase while the foot is in contact with the ground. Within the swing phase, however, there is no contact between the foot and the ground. The lower part of Figure 4 shows the horizontal $\left(F_{H}\right)$ and vertical $\left(F_{V}\right)$ ground reaction force, based on the average measuring results, presented in [12]-[18]. The upper part (right) shows a model of the location of the ground reaction force on the foot depending on the gait cycle which was used for the simulations in this contribution. The point of application of the force vector depends on the gait phase. To be able to implement the ground reaction force in the simulation model, it is modeled as a superposition of the three vectors at the points of application ( $a_{1}-a_{3}$, Figure 4(b). The according vectors are weighted by the time dependent variables $a_{1}(t), a_{2}(t)$, and $a_{3}(t)$. The sum of these variables is 1 at any time:

$$
\boldsymbol{F}_{B}(t)=a_{1}(t) \cdot \boldsymbol{F}_{B}(t)+a_{2}(t) \cdot \boldsymbol{F}_{B}(t)+a_{3}(t) \cdot \boldsymbol{F}_{B}(t)=F_{H} \cdot \boldsymbol{e}_{x}+F_{V} \cdot \boldsymbol{e}_{y}
$$

At the beginning of the stance phase the heel has the first contact with the ground: $a_{1}$ is $1, a_{2}$ and $a_{3}$ are equal to zero. Within the stance phase, the point of application moves to the toes. In this way, at the end of the stance phase, $a_{3}$ is 1 and $a_{1}$ and $a_{2}$ are equal to zero (Figure 4(a)).

With the knowledge of the external influences within a gait cycle a control scheme is developed (Figure 5). The knee mechanism, presented in this paper, damps the leg movement hydraulically using proportional valves. As a control algorithm a fuzzy controller with 25 fuzzy rules is used to calculate the optimal damping value $D_{\varphi}$ depending on the gait phase and optimized by the optimization algorithm "active set" [19] [20]. The input values of the fuzzy controller are the pressures inside the knee mechanism $\left(P_{1}\right.$ and $\left.P_{2}\right)$, as well as the knee angle $\varphi$. It is necessary to convert the necessary damping value $D_{\varphi}$ from the controller to the resulting damping values of the pistons. For this purpose, the direct kinematic is defined analytically according to the underlying geometric relations. The damping of the pistons is realized by adjusting the values of the hydraulic resistances $\left(R_{1}\right.$ and $R_{2}$ ) of the valves 1 and 2 .

The calculation of the knee angle $\varphi$ from the measured piston positions is based on the inverse kinematic, which is, however, very complex to deduce analytically. Instead, discrete values of the inverse kinematic are identified using the CAD Software ProEngineer ${ }^{\odot}$ and approximated by a fourth order Fourier transformation

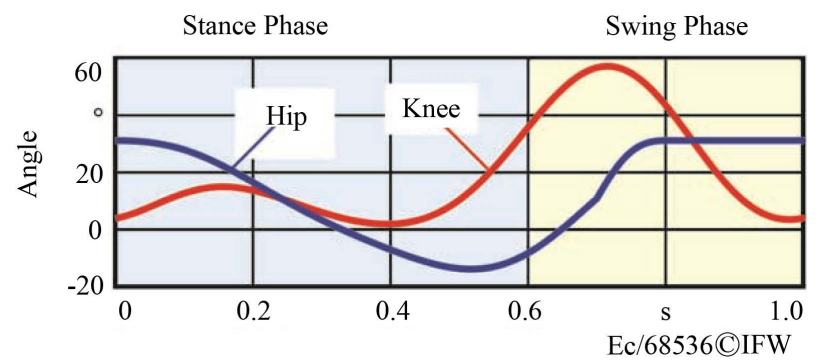

Figure 3. Pattern of movement. (a) Curve of the knee angle; (b) Curve of the hip angle. 


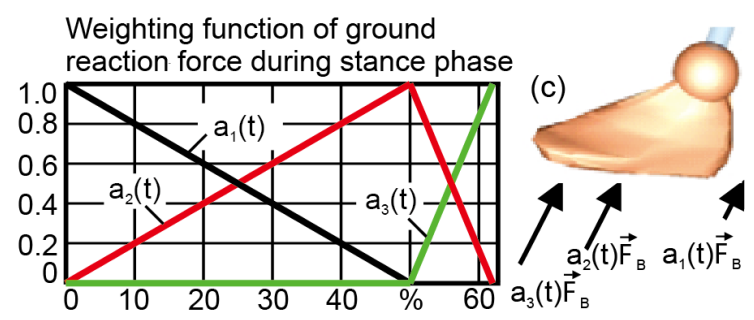

(a)

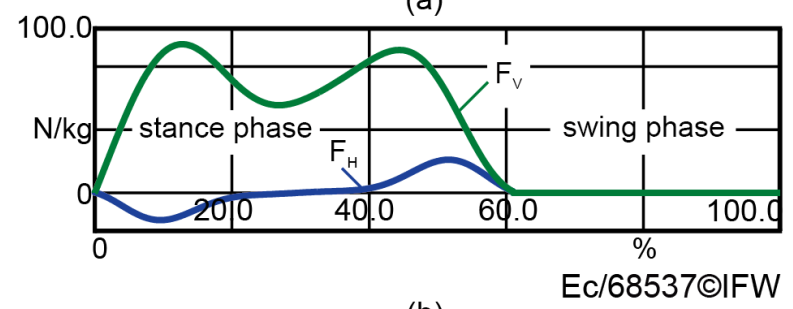

(b)

Figure 4. Kinetic influence of the walking cycle. (a) Weighting of the points of action; (b) Locations of the points of action; (c) Force curves within one gait cycle.

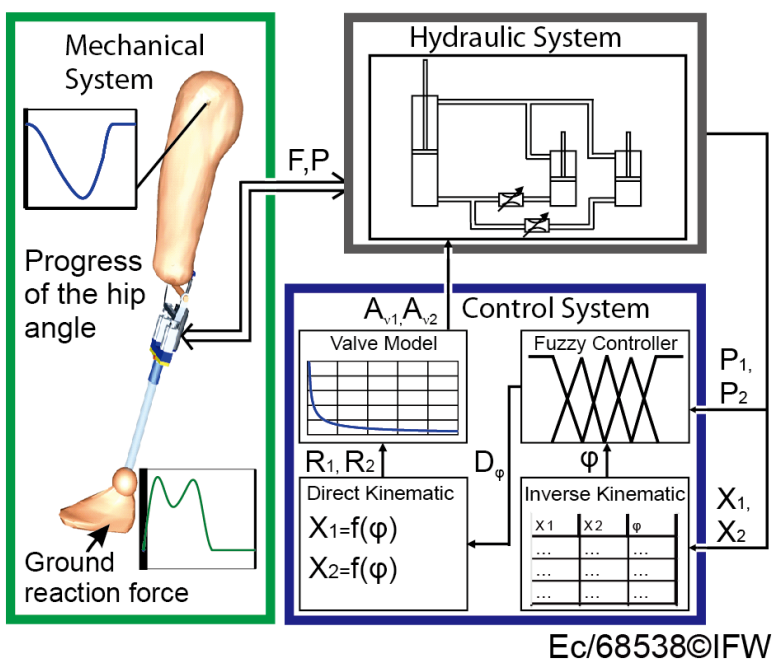

Figure 5. Control loop of the knee mechanism.

[21]. The calculation of the cross section of the proportional valves $A_{v}$ from the damping values of the pistons is derived in [22].

$$
A_{v}=\sqrt[3]{\frac{\pi \rho^{2} \mathrm{Re}_{\mathrm{krit}}^{2} v^{2}}{16 \alpha_{\mathrm{Dr}}^{4} R^{2}}}
$$

$\operatorname{Re}_{\text {krit }}$ is the critical Reynolds number $(\mathrm{Re}=2300) . v$ is the fluid kinematic viscosity, $\rho$ is the fluid density and $\alpha_{D r}$ is the flow coefficient [23]. To investigate the presented control scheme the knee mechanism is built up in a test rig (Figure 6).

Two additional pneumatic actuators are implemented into the rig. In this way, it is possible to apply forces to simulate the ground reaction force and so, to change the knee angle while simultaneously control the desired movement of the gait cycle. Displacement sensors inside of the cylinders of the knee mechanism measure the piston positions that allow the calculation of the knee angle. The test rig is controlled with a dSpace rapid prototyping system. Sample results of a kinematic gait cycle simulation are shown in Figure 7. The figure shows the piston movement, as well as the desired and the measured knee angle in a gait cycle. The results generated by 

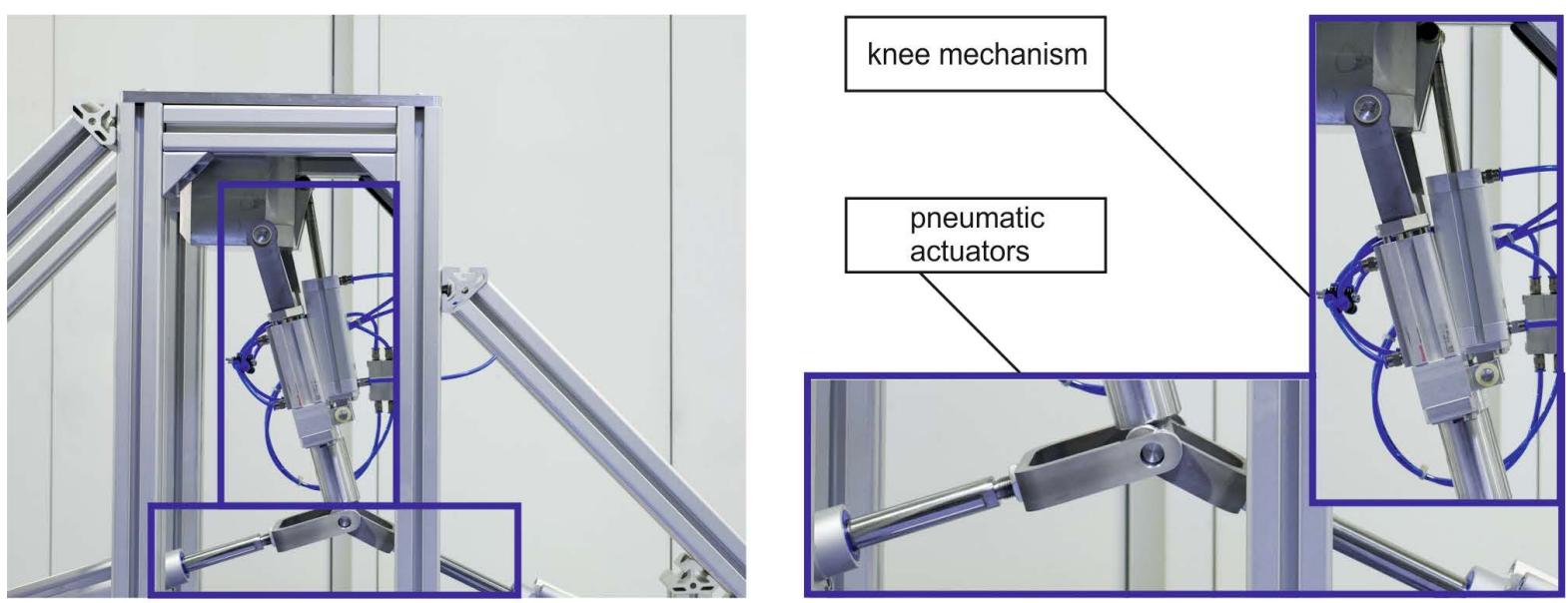

Ec/68530@IFW

Figure 6. Test bed to investigate the knee mechanism.

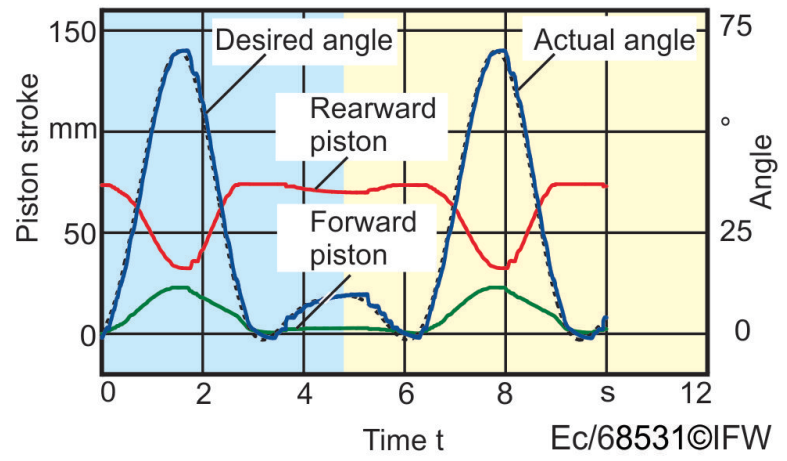

Figure 7. Results of the verifications with the test rig.

the test rig show that a gait cycle can be performed with the developed control scheme. The piston movement occurs in counter direction as expected.

\section{Compensation of Lateral Forces}

\subsection{Influence of Lateral Forces}

The patient's center of gravity is not directly above the knee joint. Thus, the displacement between the patient's center of gravity and the knee $d$ causes torque $\left(T_{\text {res }}\right)$ acting on the suspension fork of the knee mechanism (see Figure 8). This torque is amplified by long lever arms, e.g. if the patient stumbles.

The difference of the lateral forces $F_{2}$, and $F_{3}$, resulting from the torque $\left(T_{\text {res }}\right)$ leads to different movements of the pistons in the cylinders 2 and 3 and might jam those. Thus, the lateral forces must be absorbed, either mechanically or hydraulically by generating hydraulic forces which counteract the lateral forces and that way eliminate the torque $T_{\text {res }}$. The presented knee mechanism is designed to counteract the lateral forces using a linear bearing inside the hydraulic cylinders, as shown in Figure 8. The linear bearing has a height of $10 \mathrm{~mm}$, an inner diameter of $13 \mathrm{~mm}$ and the outer diameter is $17 \mathrm{~mm}$. This bearing is very light and space-saving, but, in the case of very high lateral forces, it might be too small to counteract all forces. A taller and more robust bearing would remedy this problem, but would also increase the necessary space and weight. In particular additional components outside the cylinders would have a significant stake of the prosthesis weight. Further weight reduction is attainable by combining the mechanical support structure with a new concept to reduce lateral forces hydraulically. In that case, additionally to the mechanical bearings, the hydraulic valves are controlled to generate hydraulic forces to compensate the lateral forces. The lateral forces to be compensated, are calculated as shown in (3), and (4): 


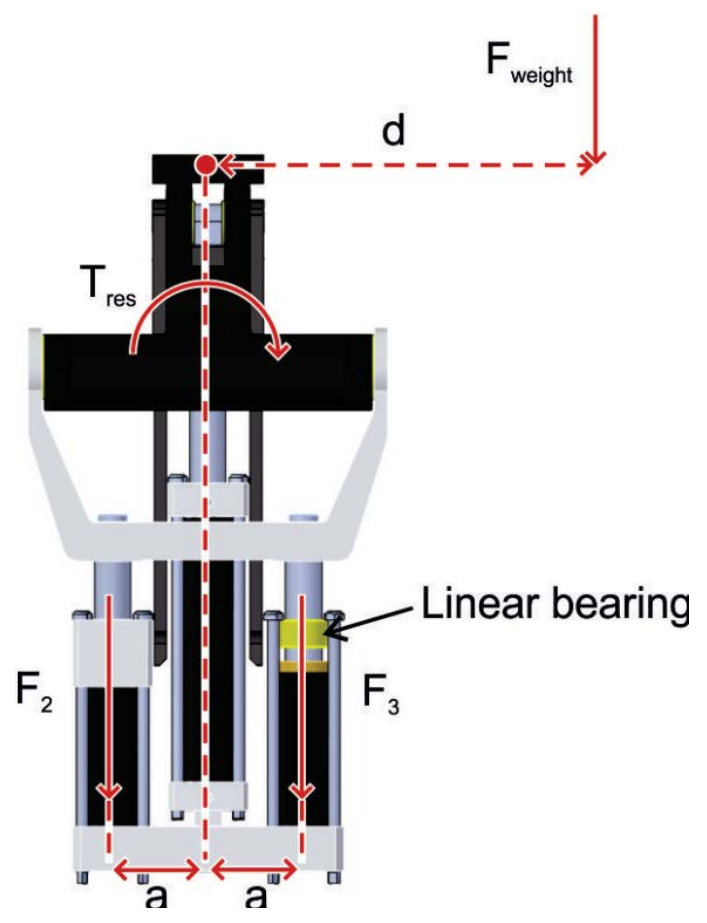

Figure 8. Force influence due to the patients weighty.

$$
\begin{gathered}
T_{\text {res }}=F_{\text {weight }} d=-F_{2} a+F_{3} a \\
F_{\text {weight }}=F_{2}+F_{3}
\end{gathered}
$$

However, since valves are passive elements, only damping forces (forces against the moving direction of the pistons) can be generated. Generating a force in moving direction, however, would require additional actuators. The necessary direction of the compensation force depends on the current load case, as shown in (5), and (6):

$$
\begin{aligned}
& F_{\text {weight }}\left(\frac{d}{a}-1\right)=-2 F_{2} \\
& F_{\text {weight }}\left(\frac{d}{a}+1\right)=2 F_{3}
\end{aligned}
$$

If the distance between the line of action of the weight force $d$ is less than the distance between the pivot of the folk and the hydraulic cylinder $a$, then both compensation forces $\left(F_{2}\right.$, and $\left.F_{3}\right)$ would act against the moving direction of the pistons and the whole torque $T_{\text {res }}$ can be eliminated by controlling the valves. If $d>a$, however, the compensation force $F_{2}$ would have to act in the same direction as the piston movement. Since a force in that direction cannot be generated in a passive system, $F_{2}$ is set to 0 and a combination of mechanical and hydraulic absorption of lateral forces is required (7).

$$
T_{\text {res }}=F_{\text {weight }} d=F_{3} a+T_{\text {mech }}
$$

$T_{\text {mech }}$ is the torque, generated by the linear bearings. To estimate the actual lever arm $d$, the standard ISO 10328 [10] is used. The different test cases expect a lever arm $d$ between $35 \mathrm{~mm}$ and $57 \mathrm{~mm}$. The distance between the pivot of the fork and the cylinder $a$ is $34 \mathrm{~mm}$. Thus, in the worst test case $(d=57 \mathrm{~mm})$, only $60 \%$ of the torque can be absorbed hydraulically and the linear bearing has to be designed to withstand $40 \%$ of the torque $T_{\text {res }}$.

\subsection{Control Based Absorption of Lateral Forces}

In the following, two approaches for a control based compensation of the lateral forces are presented. The first 
approach is an open loop control scheme (feed forward), wherein the forces are detected by force sensors and the necessary cross sections of the valves are calculated by a model of the hydraulic mechanical couplings (Figure 9(a)). The second control scheme is a closed loop control (feed back), wherein the difference of the piston position is detected and corrected by the valves (Figure 9(b)).

To realize the feed forward approach, the mathematical derivation of the mechanical and the hydraulic system in Figure 2 is presented while the mechanical system is loaded with the three forces $F_{1}, F_{2}$, and $F_{3}$.

The equations of motion of the cylinders are

$$
\begin{aligned}
& m_{1} \ddot{x}_{1}=p_{12} A_{2}-p_{1} A_{1}-F_{1} \\
& m_{2} \ddot{x}_{2}=p_{22} A_{2}-p_{1} A_{1}-F_{2} \\
& m_{2} \ddot{x}_{3}=p_{32} A_{2}-p_{1} A_{1}-F_{3}
\end{aligned}
$$

where $m_{1}$ is the mass of the piston in cylinder 1 and $m_{2}$ is the mass of the pistons in cylinders 2 and $3 . p_{1}$, $p_{12}, \quad p_{22}, \quad p_{32}$ are the pressures in the cylinder chambers (Figure 2(c)). $A_{1}$ and $A_{2}$ are the piston surfaces. The forces $F_{1}, F_{2}$, and $F_{3}$ are the loads acting on the piston rods Figure $\left.9(b)\right)$. Due to the incompressibility of the fluid, stiffness effects can be neglected. The volume flow $\dot{V}_{1}$ can be calculated as follows

$$
\dot{V}_{1}=-\dot{x}_{1} A_{1}=\dot{x}_{2} A_{1}+\dot{x}_{3} A_{1}
$$

Therefore, the relation between position velocities is

$$
-\dot{x}_{1}=\dot{x}_{2}+\dot{x}_{3}
$$

The damping effect is provided by the valves 1 and 2 . The mathematical description of the valves is analogous to a hydraulic resistor. Macia and Thaler describe a hydraulic resistor $R$ as the ratio of pressure difference $\Delta p$ and volume flow $\dot{V}$ [24].

$$
R=\frac{\Delta p}{\dot{V}}
$$

Using Equations (8)-(13) the dynamic behavior of the undamped hydraulic mechanical system can be described in the following way

$$
\ddot{x}_{1}\left(2 m_{1}+m_{2}\right)=A_{2}^{2}\left(R_{1} \dot{x}_{2}+R_{2} \dot{x}_{3}\right)-2 F_{1}+F_{2}+F_{3}
$$

Based on that general system equation, the necessary relation between the resistors $R_{1}$ and $R_{2}$ of the valves to compensate the lateral forces is derived as follows.

The pressure balance is calculated by (9) and (10):

$$
m_{2}\left(\ddot{x}_{3}-\ddot{x}_{2}\right)=A_{2}\left(p_{32}-p_{22}\right)+F_{2}-F_{3}
$$

The pressure balance

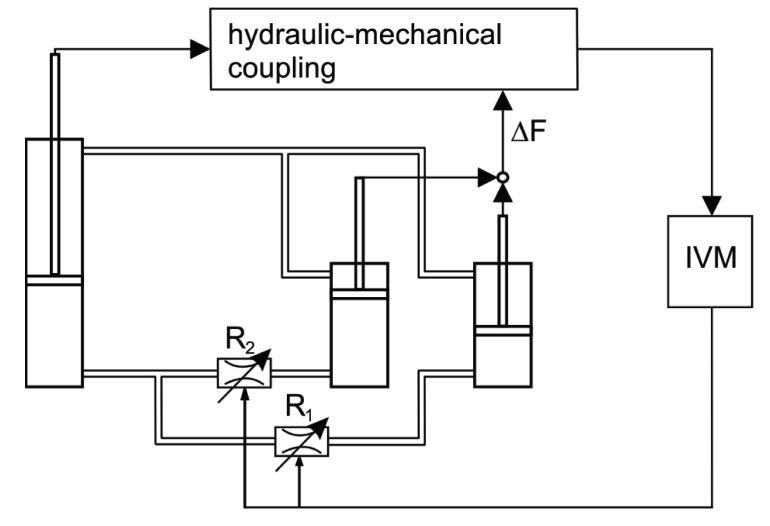

(a)

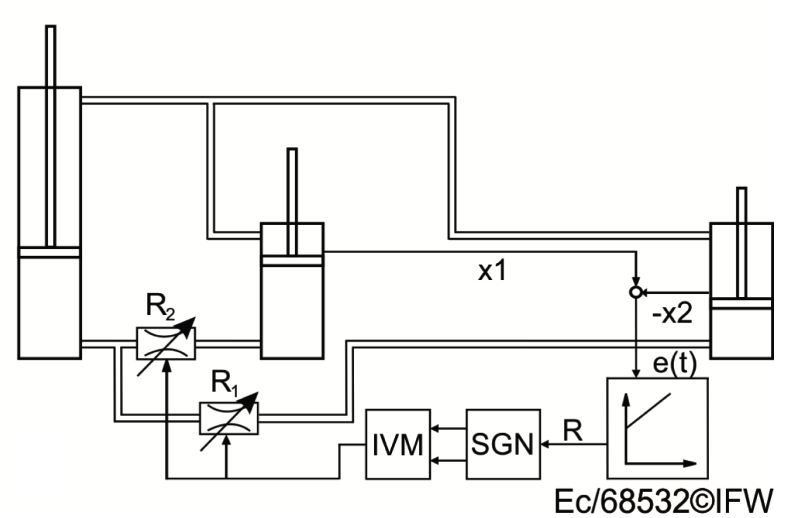

(b)

Figure 9. Control based approach to compensate lateral forces. (a) Feed forward control; (b) Feed back control. 


$$
p_{32}-p_{22}=R_{1} \dot{V}_{1}-R_{2} \dot{V}_{2}
$$

As well as (13), and (15) lead to the dynamic behavior of the description of the dynamic coupling between the pistons.

$$
\ddot{x}_{3}-\ddot{x}_{2}=\frac{1}{m_{2}}\left(A_{2}^{2}\left(R_{1} \dot{x}_{2}-R_{2} \dot{x}_{3}\right)+F_{2}-F_{3}\right)
$$

The pistons are constrained to equal speed:

$$
x_{2}=x_{3}, \quad \dot{x}_{2}=\dot{x}_{3} \quad \text { and } \quad \ddot{x}_{2}=\ddot{x}_{3}
$$

The requirement (18) as well as (12) and (17) gives the relation between $R_{1}$ and $R_{2}$, which enables the compensation of lateral forces

$$
R_{2}-R_{1}=2 \frac{F_{3}-F_{2}}{A_{2}^{2} \dot{x}_{1}}
$$

As an alternative to the feed forward approach, a feed back approach is presented, which determines the necessary cross sections of the valves to compensate lateral forces from the difference of the piston positions of cylinders 2 and 3 by a PI control element. Therefore, the positions of the piston rods of the cylinders are measured by displacement sensors and the difference of the two positions $e(t)$ is equal to the error to be compensated. Based on this error, the PI controller calculates the correcting variable $R$ to compensate the lateral forces. The demanded cross section of the valves $A_{v}$ is calculated from the correcting variable $R$ by the inverse model of the valves (IVM).

For the investigation of both approaches a Matlab/Simulink model of the knee mechanism has been developed. The mechanical part is implemented by the SimMechanics toolbox, the hydraulic part is realized by the SimHydraulics toolbox. In the simulation model the three pistons of the knee mechanism are loaded by different force steps. All force step occurs at the same time (after $0.07 \mathrm{~s}$ ).

$F_{1}$ is loaded with $1000 \mathrm{~N}, F_{2}$ is loaded $800 \mathrm{~N}$, and $F_{3}$ is loaded with $200 \mathrm{~N}$. These test signals are chosen, because they show a high difference between the lateral forces $F_{2}$ and $F_{3}$. As a result, the three pistons move differently. The mechanical coupling of the pistons of cylinder 2 and 3 via the suspension fork as well as the mechanical support structure is not considered in these investigations.

To be able to compare and evaluate the approaches to compensate lateral forces, an assessment scheme is developed. The comparison values are the error surface and the maximum error (Figure 10). The goal is to minimize both values. Figure 11 shows the results. Analyzed are the feed forward approach (FF), the feedback approach (FB) with P and PI control element as well as combinations of both.

The column chart in Figure 11(a) a shows that the feed forward approach (FF) gives very accurate results.

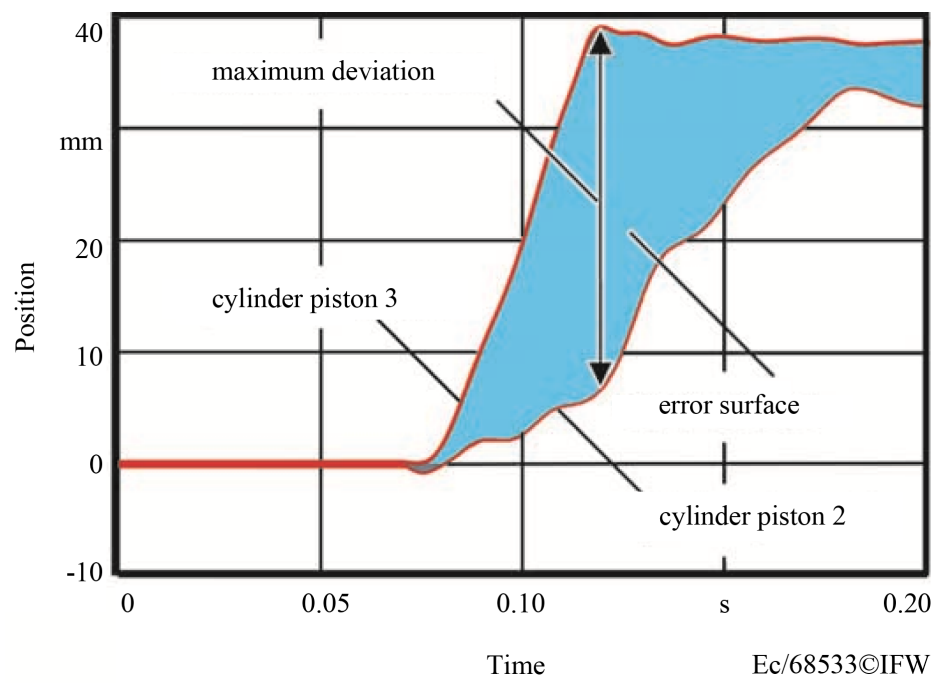

Figure 10. Assessment scheme. 


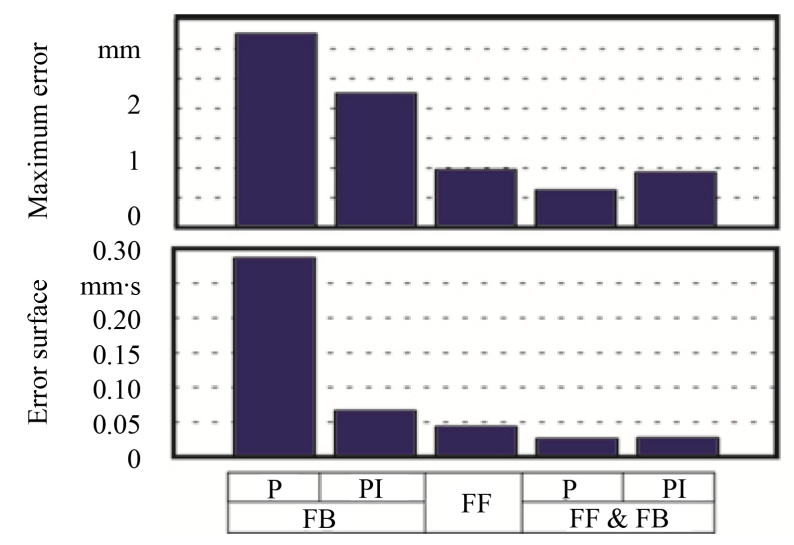

(a)

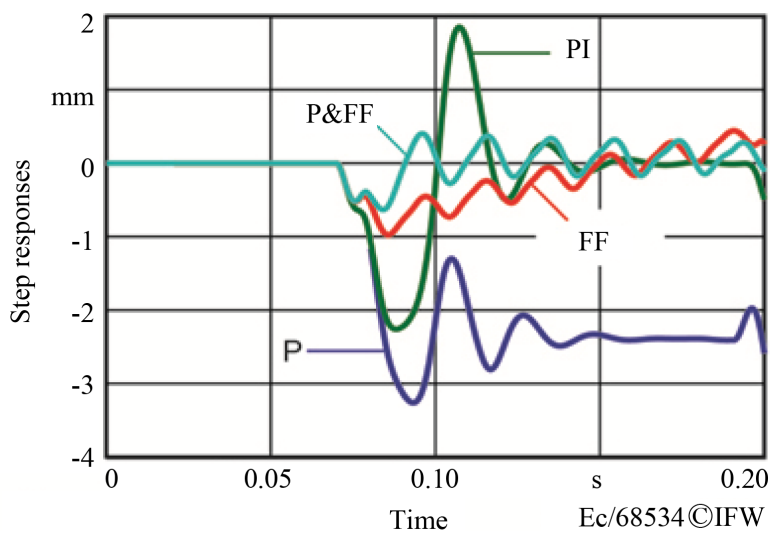

(b)

Figure 11. Results of the compensation of lateral forces.

The feed back approach (FB) based on a P-control element is less accurate. The closed loop approach based on a PI-controller starts as accurately as the P-control element which leads to almost the same maximum error (Figure 11(b)). The I-control element, however, eliminates the steady state error which leads to an error surface less than the P-control element. The best results are achieved by superposition of the open loop control and the closed loop control.

The open loop control approach shows better results for two reasons: On the one hand, the closed loop control approach because a model of the process is implemented. On the other hand, that approach includes additional process information given by the force sensors. Thus, the control is able to act before an error occurs. The closed loop control approach, however, reacts after an error has occurred which leads to higher error values. The presented investigations are based on ideal assumptions. To evaluate the actual system behavior, it is necessary to consider delays of the valves, model inaccuracies, measurement inaccuracies and inaccuracies in the valves adjustments. These evaluations are presented in Section 3.3.

\subsection{Investigation of Dynamic Influences and Interferences}

The analyses in Section 3.2 assume ideal conditions. The actual valves, however, do not act immediately but delayed. This delay is approximated by a first order low pass characteristic. Figure 12 shows the maximum deviation in response to the time constant of the valves.

For short time constants the open loop control approach shows the best results. In this case, the process model is very accurate. For higher time constants the error increases rapidly, because the model of the process does not consider the valve behavior. However, for higher time constants, the closed loop approach shows better results due to the subsequent elimination of the additional error, caused by the dynamic behavior of the valves. The best results are achieved by superposition of both approaches. Current high response valves achieve a switching frequency of $125 \mathrm{~Hz}$ and a switching time of less than $3 \mathrm{~ms}$, which is enough to generate good results as shows in Figure 12.

In summary it has been shown that the hydraulic concept is able to absorb lateral forces, if the pistons of cylinder 2, and cylinder 3 are loaded with forces with a positive sign $\left(F_{2}>0, F_{3}>0\right)$. In combination with the mechanical bearings, all loads, based on [10] can be eliminated.

\section{Active Leg Length Variation}

\subsection{Mathematical Description}

The active variation of the leg length offers advantages for the patient. After an amputation the amputee is not able to lift his foot anymore. With the active variation of the leg length the patient is able to obtain more space between the moving foot and the ground. Thus, risks of collision and stumble can be reduced. The necessary energy to change the leg length is generated by the gait cycle. Thus, no external source of energy is required.

As depicted in Section 2 the movement of the pistons inside of the forward and the rearward cylinders in op- 


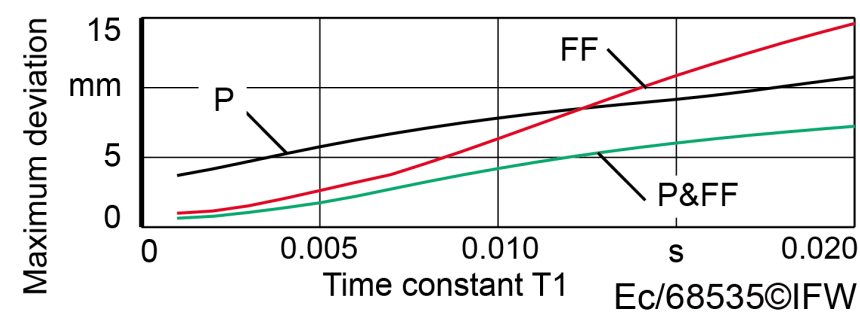

Figure 12. Influence of the valves dynamic.

posite direction changes the knee angle. Uniform movement of all three pistons, however, leads to the variation of the leg length. To enable uniform movement, the hydraulic scheme, depicted in Figure 2(c) needs to be expanded by an additional volume chamber to compensate the volume of the piston rods. The expanded hydraulic scheme is shown in Figure 13. In order to simplify the following calculations the valves 1 and 2 are summarized to the valve $b$. In that case the pressures in the chambers as well as the piston rods in the forward cylinders are equal.

This hydraulic scheme enables piston movements in opposite direction to change the knee angle as well as uniform motion to change the leg length. Superimposed motion of the angle and leg length is also possible. The directions of the movement of the pistons are adjusted by the two valves $a$ and $b$ by adjusting the cross section of the valves.

The shortening of the leg length happens in the stance phase and is driven by the weight of the patient. All three pistons move in negative direction $\left(-x_{1},-x_{2}\right)$ and the hydraulic fluid fills the chamber (compensation element). Additionally, the weight of the patient compresses a spring energy storage. The stored energy is used to extend the leg length in the end of the swing phase.

The valve $b$ damps the opposite movement of the pistons of the backward and the forward cylinders and that way it damps the knee angle, while the valve a damps the uniform motion of the cylinders and controls the leg length variation. The mathematical description of the expanded hydraulic scheme is shown as follows:

$$
\begin{gathered}
\dot{V}_{a}=A_{k} \cdot \dot{x}_{k} \\
\dot{V}_{b}=A_{2} \cdot \dot{x}_{2} \\
R_{b}=\frac{p_{1}-p_{2}}{\dot{V}_{b}} \\
R_{a}=\frac{p_{1}-p_{k}}{\dot{V}_{a}}
\end{gathered}
$$

where $R_{a}$ and $R_{b}$ are the hydraulic resistors of the valves $a$ and $b$. The necessary value of $R_{b}$ depends on the demanded motion of $\dot{x}_{2}$ and is calculated by the Equations (21)-(22)

$$
R_{b}=\frac{p_{1}-p_{2}}{A_{2} \dot{x}_{2}}
$$

The necessary value of $R_{a}$ is calculated by the Equation (20), and Equation (23) as follows:

$$
R_{a}=\frac{p_{1}-p_{2}}{A_{k} \cdot \dot{x}_{k}}
$$

The Equations (24)-(25) enable the calculation of the values of the hydraulic resistances $R_{a}$, and $R_{b}$. These values lead to the demanded damping of the knee angle and the demanded leg length variation at the same time. The relation between hydraulic resistor and the adjustable cross section of the valve as well as the relation between positions and the knee angle is depicted in Section 2.2.

\subsection{Simulation of a Gait Cycle with Active Leg Length Variation}

The scheme, presented in Figure 13 is investigated with a simulation model. The control scheme as well as the 


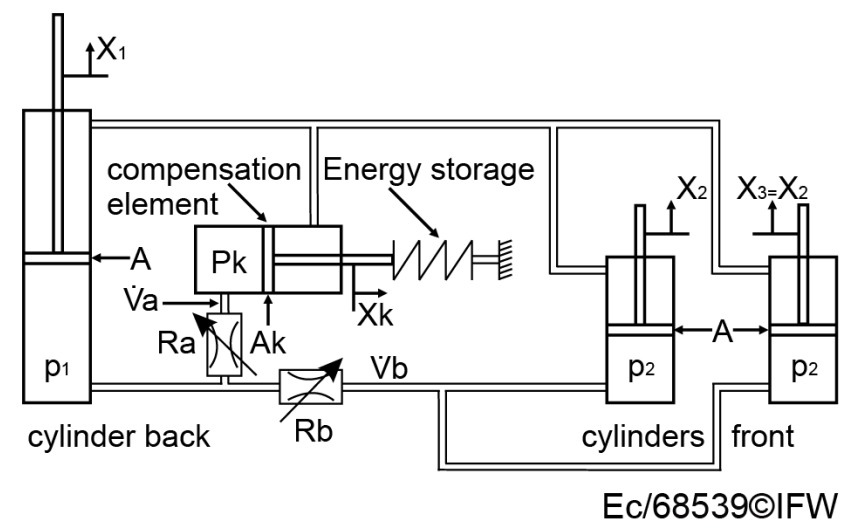

Figure 13. Extended hydraulic scheme.

inverse and direct kinematics are realized by Matlab/Simulink. This model allows simulating a whole gait cycle as depicted in Figure 14.

The actual ground reaction force and the influence of the torque from the hip are considered in this simulation model. The simulation shows that a gait cycle with active leg length variation can be realized with the presented knee mechanism. Figure 14 shows a gait cycle with leg length variation. The leg is shortened within the stance phase using the patient's weight and it is lengthened within the swing phase using the force of the spring.

In the presented case, the leg length is varied by $10 \mathrm{~mm}$. However, the curve of the leg length is just an example to show, that the actual leg length follows the desired leg length very well. The necessary curve of leg length variation is an anatomic challenge which has not been investigated yet.

Alternative to the presented hydraulic scheme, it is possible to realize leg length variation without an additional energy store. In that case, within the stance phase, only the piston inside the backward cylinder is moving and that way the knee angle as well as the leg length is changing, while a volume chamber is filled. To extend the leg length within the swing phase, the kinetic energy of the leg is used.

\subsection{Investigation of Dynamic Influences}

The presented simulation assumes ideal conditions. Additionally, the influence of the low pass behavior of the valves is investigated using the assessment scheme, presented in Section 3.2. Here, the influence of the accuracy of the leg length variation of $10 \mathrm{~mm}$ is explored. The knee angle is not considered because the leg length variation is more sensitive than the knee angle damping.

Figure 15 shows the influence of the low pass behavior. The error of the leg length increases approximately linearly. A time constant of 0.02 seconds leads to an error of the leg length of $1 \mathrm{~mm}$ or $10 \%$ of the desired variation.

In general the influence of the valve behavior of the gait cycle as well as of the leg length variation is less than of the compensation of the lateral forces, because the lateral forces appear immediately.

\section{Conclusions}

This paper provides an overview into the development of a novel mechanism for knee disarticulation prostheses. Common approaches of artificial knee joints consist of a mechanical support structure to transfer forces and an additional hydraulic damping element. In contrast, for this approach, no mechanical support structure is necessary. Instead, the hydraulic system is designed to transfer the weight force, as well as to reduce the lateral forces. This makes the mechanism more flexible; it helps to reduce weight and allows additional applications, like the active variation of the leg length within a gait cycle. First, a method to compensate lateral forces hydraulically is presented. Here the control of the joint mechanism is augmented with a mathematical model for the description of the mechanical hydraulic coupling. Via a controlled operation of the valves the lateral forces are absorbed by the hydraulic system. Furthermore, a control scheme is presented which enables the active variation of the leg length with this novel knee mechanism. The patient's weight force is used to shorten the leg length in the stance phase. Hereby a spring is compressed. Using the spring energy, a leg elongation in the swing phase is achieved. 


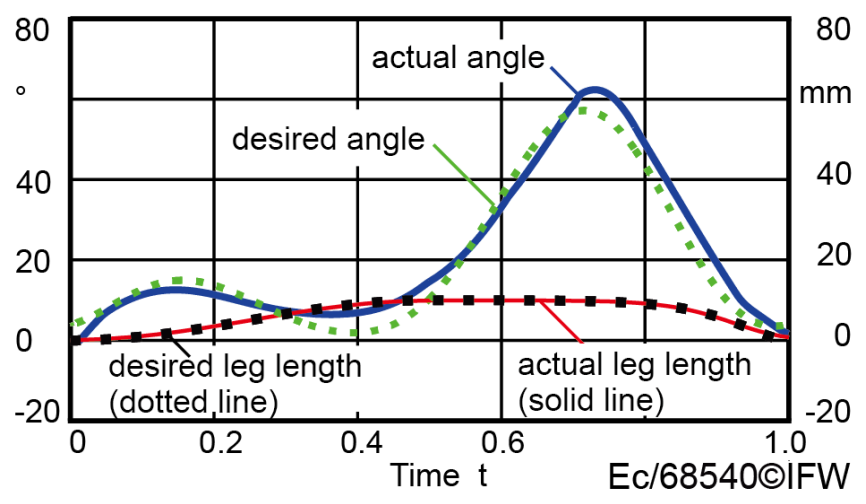

Figure 14. Simulation of a gait cycle with leg length variation.

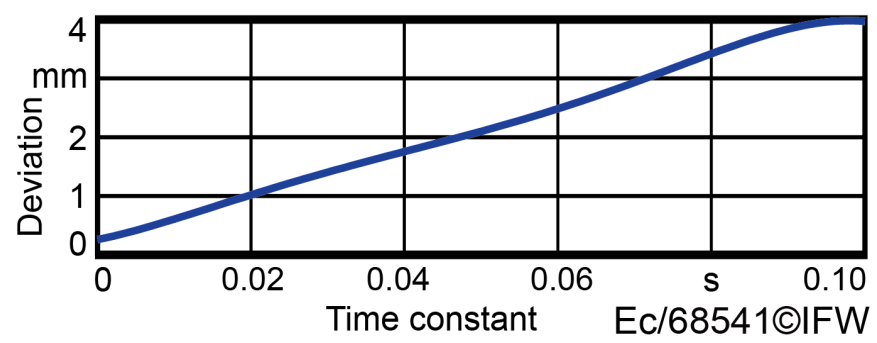

Figure 15. Influence of the valve dynamic of the leg length variation.

Since the only source of energy is the weight force, a leg length variation of $20 \mathrm{~mm}$ can be realized without additional actuators. By using the kinetic energy in the swing phase, it is also possible to realize active leg length variation without an additional spring energy store.

With the new prosthesis, a knee angle of $136^{\circ}$ is attainable and it is designed to withstand a patient's weight of $125 \mathrm{~kg}$. The prosthesis complies with the standard ISO10328 and weighs only $2.5 \mathrm{~kg}$.

\section{Acknowledgements}

The research studies, presented in this paper, have been part of the cooperation project MultiPro. The authors would like to thank the Federal Ministry for Research and Education (BMBF) for funding this project.

\section{References}

[1] Hagberg, E., Berlin, Ö.K. and Renström, P. (1992) Function after Through-Knee Compared with Below-Knee and Above-Knee Amputation. Prosthetics and Orthotics International, 16, 168-173.

[2] Behr, J., Friedly, J., Molton, I., Morgenrot, D., Jensen, M.P. and Smith, D.G. (2009) Pain and Pain-Related Interference in Adults with Lower-Limb Amputation: Comparison of Knee-Disarticulation, Transtibial and Transfemoral Surgical Sites. Journal of Rehabilitation \& Development, 46, 963-972. http://dx.doi.org/10.1682/JRRD.2008.07.0085

[3] Greitemann, B. (2005) Kniegelenknahe Amputationen. In: Wirth, J.C. and Zichner, L., Eds., Orthopädie und Orthopädische Chirurgie, Georg Thieme Verlag, Stuttgart, 481-491.

[4] Baumgartner, R.F. (1979) Knee Disarticulation versus Above-Knee Amputation. Prosthetics and Orthotics International, 3, 15-19.

[5] Mensch, G. (1983) Physiotherapy Following Through-Knee Amputation. Prosthetics and Orthotics International, 7, 79-87.

[6] Stark, G. (2004) Overview of Knee Disarticulation. American Academy of Orthotists \& Prosthetists, 16, 130-137.

[7] O’Connor, R.S. (1999) Prosthesis for Long Femur and Knee Disarticulation Amputation. Patent 5895430, USA.

[8] Kramer, S., Srinivasan, S. and Swanson, V. (1998) Knee Joint Mechanism for Knee Disarticulation Prosthesis. Patent 5746774, USA.

[9] Denkena, B., Simon, S. and Zager, H. (2011) Knee Exarticulation Prosthesis for Use as Hydraulic Knee Joint Prosthe- 
sis for Human Leg, Has Hydraulic Assemblies Causing Motion of Artificial Replacement Member of Leg Sections around Rotary Axis by Movement in Opponent Arrangement. Patent DE102009056074A1, Germany.

[10] ISO10328:2006 (2006) Prosthetics-Structural Testing of Lower-Limb Prostheses-Requirements and Test Methods.

[11] Perry, J. and Burnfield, J. (1992) Gait Analysis: Normal and Pathological Function. Slack, New York.

[12] Whittle, M.W. (1996) Gait Analysis: An Introduction. Butterworth-Heinemann Ltd., Oxford.

[13] Al-Hamadi, A., Andres, S., Berndt, D., Calow, R., Lehmann, C., Michaelis, B., Schünemann, S. and Urbansky, U. (2004) 3D-Bewegungsanalyse in der "Neuro-Medizintechnik". Ph.D. Dissertation, Otto-von-Guericke-Universität Magdeburg, Magdeburg.

[14] Götze, C., Sippel, C., Rosenbaum, D., Heckenberg, L. and Steinbeck, J. (2003) Objective Measures of Gait Following Revision Hip Arthroplasty. First Medium-Term Results 2.6 Years after Surgery. Zeitschrift für Orthopädie und ihre Grenzgebiete, 141, 201-208.

[15] Seichert, N., Erhart, P. and Senn, E. (1997) Die Etablierung der instrumentierten Ganganalyse (IGA) als Verfahren zur unmittelbaren klinikrelevanten Gangbeurteilung. Darstellung der propulsiven und bremsenden Muskelaktivitäten beim Gehen. Physikalische Medizin, Rehabilitationsmedizin, 7, 1-11. http://dx.doi.org/10.1055/s-2008-1061850

[16] Kramers-de Quervain, I.A., Stüssi, E. and Stacoff, A. (2008) Ganganalyse beim Gehen und Laufen. Schweizerische Zeitschrift für Sportmedizin und Sporttraumatologie, 56, 35-42.

[17] Leuchte, S. and Luchs, A. (2006) How Symmetrical or Asymmetrical Is the Physiological Gait in Respect of Age? Journal of Physical and Rehabilitation Medicine, 16, 96-102.

[18] Hölscher, C. (2009) Auswirkungen Von Hohen Tibialen Umstellungs-Osteotomien auf den Gang und die KniegelenksBelastung. Ph.D. Dissertation, Medizinische Fakultät der Westfälischen Wilhelms-Universität Münster, Münster.

[19] Fletcher, R. (1987) Practical Methods of Optimization. John Wiley \& Sons Limited, West Sussex.

[20] Gill, P.E., Murray, W. and Wright, M. (1982) Practical Optimization. Emerald Group Publishing Limited, London.

[21] Sneddon, I.N. (1995) Fourier Transforms. Dover Publications, Inc., New York.

[22] Clarke, J.A. (1985) Energy Simulation in Building Design. Butterwort-Heinemann, Oxford.

[23] Crane Co., NY. (1982) Flow of Fluids through Valves, Fittings and Pipes. Metric Edition-SI Units, Technical Paper No. 410M, Crane Co., New York.

[24] Macia, N.F. and Thaler, G.J. (2005) Fluid Systems-Hydraulic. In: Macia, N.F. and Thaler, G.J., Eds., Modeling \& Control of Dynamic Systems, Thomson Delmar Learning, Clifton Park, 91-95. 
Scientific Research Publishing (SCIRP) is one of the largest Open Access journal publishers. It is currently publishing more than 200 open access, online, peer-reviewed journals covering a wide range of academic disciplines. SCIRP serves the worldwide academic communities and contributes to the progress and application of science with its publication.

Other selected journals from SCIRP are listed as below. Submit your manuscript to us via either submit@scirp.org or Online Submission Portal.
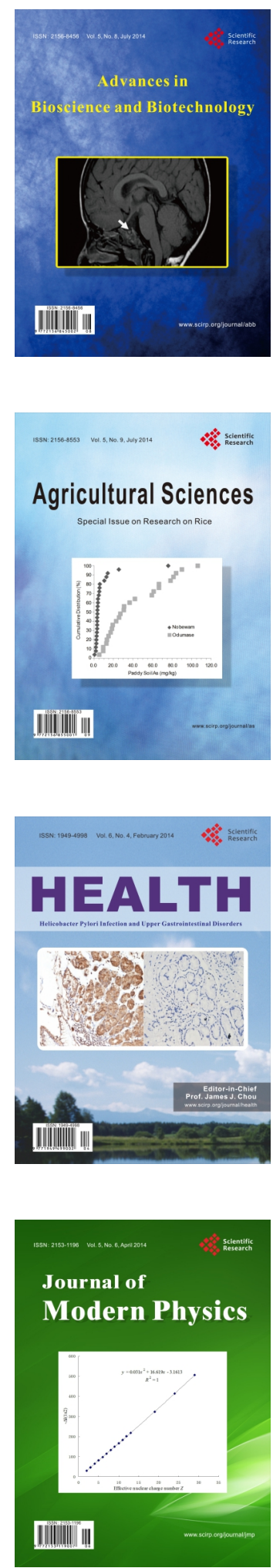
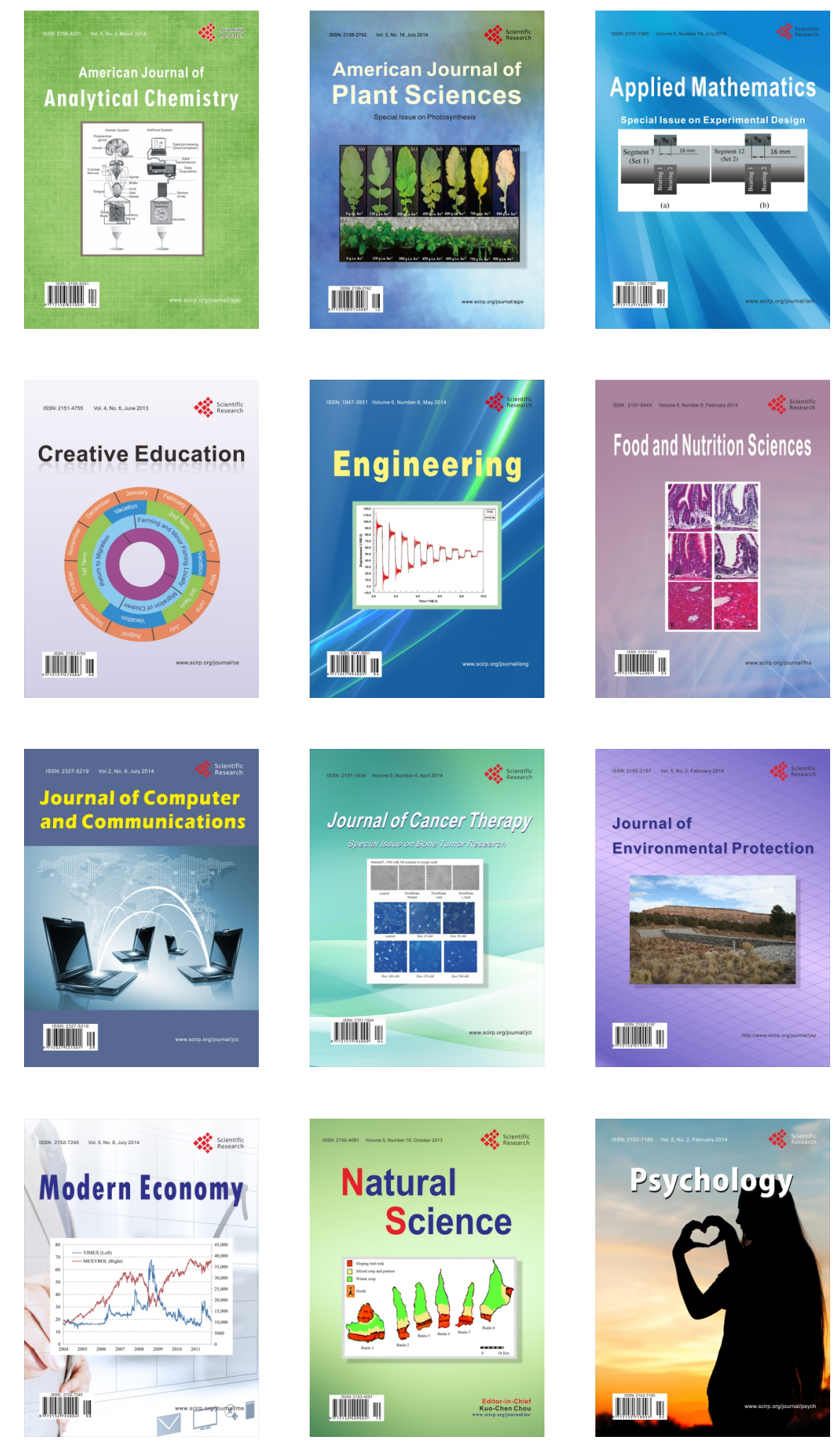